\title{
BMJ Open Preferences for end-of-life care: a study protocol for a cross-sectional survey of Chinese frail elderly home residents in Hong Kong
}

Bo Yan (D) , ${ }^{1}$ Xinyi Xu, ${ }^{1}$ Patsy PH Chau, ${ }^{1}$ Naomi Takemura, ${ }^{1}$ Derek YT Cheung (D),${ }^{1}$ Felix HW Chan, ${ }^{2}$ Chia-Chin Lin ${ }^{1,3}$

To cite: Yan B, Xu X, Chau PPH, et al. Preferences for end-of-life care: a study protocol for a crosssectional survey of Chinese frail elderly home residents in Hong Kong. BMJ Open 2020;10:e033862. doi:10.1136/ bmjopen-2019-033862

- Prepublication history for this paper is available online. To view these files, please visit the journal online (http://dx.doi. org/10.1136/bmjopen-2019033862).

Received 25 August 2019 Revised 04 March 2020 Accepted 05 March 2020
Check for updates

(C) Author(s) (or their employer(s)) 2020. Re-use permitted under CC BY-NC. No commercial re-use. See rights and permissions. Published by BMJ.

${ }^{1}$ School of Nursing, LKS Faculty of Medicine, University of Hong Kong, Hong Kong SAR, China

${ }^{2}$ Department of Medicine, LKS Faculty of Medicine, University of Hong Kong, Hong Kong SAR, China

${ }^{3}$ School of Nursing, College of Nursing, Taipei Medical University, Taipei, Taiwan

Correspondence to Professor Chia-Chin Lin; cclin@hku.hk

\section{ABSTRACT}

Introduction Existing literature on attitudes toward endof-life care (EoLC) covers the general public but has little information on the frail elderly population. The aim of the current study is to investigate the preferences of Chinese frail elderly home residents with respect to EoLC by conducting cross-sectional surveys.

Methods and analysis Surveys, including resident and family versions, were developed based on the existing literature and our pilot interviews. The targeted participants were 400 frail elderly home residents (aged $\geq 65$ years old) and 200 family caregivers. Purposive sampling will be used as each elderly home will help to recruit five to 15 elderly participants for the study. Descriptive analysis and modelling will be used to examine preferences on EoLC and related factors, as well as to compare the responses of elderly home residents with those of their family caregivers.

Ethics and dissemination The cross-sectional survey has obtained approval from the Institutional Review Board. Confidentiality and safety issues will be carefully observed. The results of the study will be disseminated through international conferences, peer-reviewed academic journal publications, and a report in plain language to be shared with elderly residential homes.

\section{INTRODUCTION}

End-of-life care (EoLC) attitudes have increasingly attracted attention in peer-reviewed journals. ${ }^{1-4}$ The literature indicates that EoLC for terminally ill patients can include many challenges, for example, access to services, quality of care, awareness, attitudes, communication and decision making. ${ }^{4}$ Advance care planning (ACP) and advance directives (ADs) have been promoted in order to discover patient attitudes towards their EoLC, ${ }^{5}$ with the benevolent intention that these processes help to honour their wishes or preferences for a good death. An AD is one of the potential outcomes of participation in ACP. Both ADs and ACP involve the patients' understanding of life-sustaining treatment (LST) ${ }^{6}$ Several studies have demonstrated that the
Strengths and limitations of this study

- Survey versions were developed to inquire into the preference of both the frail elderly home residents and family caregivers.

- For the first time, surveys on elderly people's attitudes towards end-of-life care cover all main areas of Hong Kong.

- The cross-sectional design of this study cannot conclude causality.

- Participants with communication problems (ie, deafness or aphasia) were excluded due to the nature of the data collection method.

awareness of $\mathrm{AD}$ is low in Chinese populations. ${ }^{1-35}$ Moreover, a recent systematic review summarised 107 EoLC studies in Hong Kong in the past decade reported a primary focus on AD rather than ACP. ${ }^{4}$ Studies of Chinese family attitudes towards ACP present mixed findings according to Lee's review in $2014 .^{5}$ Therefore, our study aims to investigate the preferences of Chinese frail elderly home residents with respect to EoLC. We explore their preferences along with relevant factors, such as ACP and AD awareness, preferences concerning LST, decision-making,and preferred place of death, by conducting original surveys with frail elderly home residents and their family caregivers.

By definition, frailty refers to a vulnerable state in which there is an increased risk of hospitalisation, deterioration in functioning, and mortality. ${ }^{7}$ Frail elderly people are particularly understudied, and thus their voices have rarely been heard by academics, politicians, or the wider public. Though we found the most recent report on the prevalence of Hong Kong general public preferences regarding EoLC and preferred place of death, ${ }^{8}$ these results were generated from telephone landline surveys. The public 
participants who joined the brief phone interview may not represent the views of frail elderly home residents on these sensitive topics. The landline phone calls could not reach the frail elderly population living in residential care homes for the elderly (RCHEs), including nursing homes, care and attention homes, and other types of homes that provide comprehensive care for dependent people. ${ }^{9}$ Our study will help to bridge this knowledge gap. Face-to-face interviews with frail elderly home residents will help to gain a better understanding of their attitudes.

The present study is significant as the population of Hong Kong ranks top in life expectancy around the globe, and the percentage of its ageing population is rising. ${ }^{19}$ The government officially projects that the percentage of elderly residents aged 65 and above will continue to grow from over one million to 2.61 million in 2058 (36\% of the Hong Kong population). ${ }^{10}$ This is much higher than estimates of the global elderly population $(21.1 \%$ in 2050). ${ }^{11}$ At present, the demands for residential elderly care services in Hong Kong remain overwhelming, with average waiting time as long as 24 months for admission. ${ }^{12}$ Due to priority considerations, frailty is one of the primary characteristics taken into account for elderly people being admitted to Hong Kong RCHEs. ${ }^{9}$ Therefore, it is critically important to study the elderly home residents with pre-frail or frail statuses as they are most likely to receive EoLC in residential homes. ${ }^{9}$

Meanwhile, knowledge about the region must be urgently updated as the only studies on EoLC among elderly home residents in Hong Kong were conducted roughly 10 years ago. ${ }^{4612}$ Hypothesising those factors that influence their preferences from the existing literature enables us to explore additional possible predictors. The present study will use a comprehensive design to explore the under-researched topic of EoLC preferences of an understudied population: frail elderly home residents. Within families, caregivers' attitudes are also crucial, as they play a significant role as surrogate decision-makers in the future circumstance of an elderly family member, who does not have an $\mathrm{AD}$ or ACP, losing the capacity to make decisions on their own. Families often find making EoLC decisions very stressful. ${ }^{13}{ }^{14}$ The systematic review by Wang and Chan shows that family caregivers in Hong Kong have also been an insufficiently studied population. ${ }^{4}$ Thus, it is worth further investigation of caregiver's attitudes, and so we include this comparative perspective in our analysis. This is consistent with the WHO's initiative to build an age-friendly environment. ${ }^{11}$ To enhance the quality of EoLC in RCHEs, our survey findings will be a helpful reference to further identifying some directions for public education and other interventions in order to meet the needs of Hong Kong's frail elderly home residents.

\section{Research questions}

Phase 1: Survey of elderly home residents

What is the level of awareness of such terms as living will, $\mathrm{AD}$, or ACP in Hong Kong among the frail elderly people, and what are their preferences with regard to ACP, decision-making, LST, AD, EoLC and preferred place of death?

\section{Phase 2: Family caregiver survey}

What is the level of awareness of such terms such as living will, AD, or ACP in Hong Kong among family caregivers, and what are their attitudes of elderly family members' preferences?

\section{METHODS AND ANALYSIS Design}

Prior to the main cross-sectional study, a pilot questionnaire has been completed on one site with a sample of 12 participants in an RCHE. The feasibility of the survey content was assessed. During our pilot survey interviews, we found the majority (10 out of 12) of participants who agreed to join the study were happy or felt it was acceptable to discuss EoLC issues with us, while the remaining two participants lost interest or refused to continue in the middle of the interviews, and their withdrawal was respected. Afterwards, no severe distress was reported or observed. Similarly, the existing literature shows that elderly people in Hong Kong facilities are willing to talk about issues related to death and dying. ${ }^{12}$ This offers our survey the advantage of gaining first-hand knowledge about the elderly people's preferences and experiences. In the pilot, only one participant out of the 12 participants had heard of $\mathrm{AD}$ or ACP before our interviews. This low level of awareness is consistent with earlier surveys that reported that $96 \%$ and $85.7 \%$ of people surveyed had never heard of $\mathrm{AD} .{ }^{18}$

We conducted four phone interviews with family caregivers as a pilot for the family version of the survey. Three of the four interviews were completed, and the only dropout occurred part way through an interview when the participant incorrectly thought that our interview was an attempt to sell her something. Feasibility and acceptance of our survey appear high among frail elderly home residents and family caregivers in Hong Kong. We have made modifications to the version for the elderly person to shorten the questionnaire to reduce the burden of participation. We have added explanations of ACP and $\mathrm{AD}$ in plain language so that it is easier for lay participants to understand. We have provided more choices on why participants do or do not want to consider initiating ACP or an $\mathrm{AD}$ based on the pilot findings.

\section{Outcomes}

i. Phase 1 Primary outcome: Preferences of the frail elderly person on ACP.

ii. Phase 1 Secondary outcomes: The frail elderly person's level of awareness and preferences with regard to LST, decision-making, $\mathrm{AD}$, preferred place of death and quality of life.

iii. Phase 2 Primary outcome: Level of agreement between Family caregivers' preferences and those of elderly's on ACP for elderly home residents. 
iv. Phase 2 Secondary outcomes: Family caregivers' awareness of these terms and preferences with regard to LST, decision-making, ACP, AD, and preferred place of death.

\section{Participants}

Phase 1: Frail elderly home residents

The inclusion criteria for the frail elderly participants are as follows.

i. At least 65 years of age.

ii. Chinese in elderly homes who can speak and understand either Cantonese or Mandarin.

iii. Either pre-frail or frail status (rating 1-5 on FRAIL scale (Fatigue, Resistance, Ambulation, Illnesses, \& Loss of Weight) ). ${ }^{15}$

The exclusion criteria for frail elderly participants are:

i. Cognitive impairment (scoring 5 or below on the abbreviated mental test (AMT) ). ${ }^{16}$ To screen for ineligibility due to cognitive impairment, the Chinese AMT will be used.

ii. Communication problems due to functional impairment (for example, deafness or aphasia).

\section{Phase 2: Family caregivers}

The inclusion criteria for family caregiver participants are as follows.

i. Hong Kong Chinese adults (18 years of age or above) who can speak and understand Cantonese or Mandarin.

The exclusion criteria for family caregiver participants are:

i. Communication problems due to functional (deafness or aphasia) or intellectual impairment.

\section{Recruitment}

We plan to partner with 32 local elderly homes with an average of 12 residents from each home. Under the most conservative scenario (assuming the true proportion is $50 \%$ ), a sample of 384 elderly residents will be required to give a $95 \%$ CI for the proportion of people who would prefer to have communication on ACP with a margin of error of $5 \%$. To cater for inter-correlation within residents in the same home, we assume an Intraclass Correlation Coefficient (ICC) to be 0.05 . We will expect to interview 400 elderly participants in order to collect their responses to the survey in the first phase. Assuming that around half of these elderly people will have family caregivers willing to join the study, 200 family caregivers will be interviewed in the second phase.

Using a purposive sampling method, we plan to select elderly homes in all main regions of Hong Kong (Hong Kong Island, Kowloon, and the New Territories) by sending out invitations via mail and by convenient professional networks. The managers of the elderly care homes will be approached via phone calls and asked permission to let us interview their elderly residents. The elderly residents in the homes will be invited in-person to participate in face-to-face survey interviews. The family caregivers can choose to join caregiver interviews via either face-toface or via phone survey with our research staff at a time convenient as mutually agreed.

The facility staff will introduce us to the elderly candidates. There will be no monetary incentives offered to either the participants or the agencies involved. During the recruitment process, the research assistant (RA) will explain the study to the potential participants either in a private room (bedside/chairside) or in the corner of a common room to protect privacy. After seeking oral consent from residents to participate, the RA will administer the AMT and the five-item Frailty scale to screen out ineligible ones based on our exclusion criteria. The RA will then conduct an individual face-to-face interview with the participant using our designed questionnaire. Trained RAs will collect data during the interviews on either paper or digital form, depending on Internet availability. Digital devices (ie, smartphones or tablets) for accessing the digital survey will be operated by the research staff during the interviews with the participants, and paper-based surveys will serve as a backup option.

\section{Survey interviews}

A structured questionnaire (one version for the frail elderly and the other version for their family caregivers) has been developed based on the objectives of the current study and with reference to a recent publication on similar topics. ${ }^{1-3}$ Additional valid scales from existing evidence were integrated to measure important factors (for example, geriatric depressive symptoms ${ }^{17}$ ) that could potentially affect participants' EoLC attitude in this comprehensive survey study. The authors ascertained face validity based on literature and research experience in the area. The Chinese questionnaires have been viewed by a panel of six Hong Kong local experts, including one geriatric doctor, one clinical psychologist, two nurses and two social workers. The first elderly questionnaire has been pilot tested on one site with a sample of 12 participants to test for feasibility. We then revised the survey in preparation for the main study. After the pilot test, the experts were invited to judge the content validity of the questions for the second time as the questionnaires were developed. The content validity index (CVI) was calculated and considered as excellent (Average CVI=0.87 for elderly survey and 0.98 for caregiver survey).

\section{Phase 1 measurement}

The AMT and FRAIL screening tools will be applied to select our participants. The interview for the frail elderly participants will be conducted if they are deemed eligible. The questionnaire contains seven parts: (A) Demographics and functioning (personal profile). (B) Healthcare experiences and attitude. (C) Expectations of EoLC. (D) Advanced Care Planning experience and attitude. (E) Advance Directives awareness, experience, and attitude. (F) Ideal terminal/death environment (preferences on places of care and eventual death); and $(G)$ quality of Life. 
A. Demographic information including age, sex, educational level, marital status, religion, financial source, type of nursing home, family members, self-reported health and pain severity, and bereavement experience, etc, will be collected. To assess daily functioning, frailty, depressive symptoms and suicidal ideation, we will use the following respective scales, 10-item Barthel Index on ADL, ${ }^{18} 5$-item FRAIL, ${ }^{15}$ Geriatric Depression Scale GDS-4, and a single-item suicidal ideation assessment. ${ }^{19}$

B. The participants will be asked to share their experience of and attitude toward life-sustaining treatment and previous hospitalisation according to questions we have devised.

C. The participants will be asked to rate their opinions on decision making with regard to LST in a hypothetical dying scenario, and their attitudes on quality of life at the end of life. The hypothetical dying scenario will be described in one phrase as 'becoming seriously ill but with no cure available' for themselves.

D. The participants will be asked about their awareness and experience or participation in ACP, their attitudes and preferred communication with others, as well as their reasons for rejecting the idea of ACP.

E. Participants will be asked about their awareness of a living will and $\mathrm{AD}$, and about their current consideration in formulating their written preferences on medical decisions and $\mathrm{AD}$. The reasons behind their choices and the persons who introduced these topics to them will also be explored.

F. The participants will be asked to rate their opinions on their hypothetical dying situation, that is, the ideal places of their terminal care and death. They will be asked about their preferences regarding dying alone or having certain persons around them during their own eventual death.

G. The McGill Quality of Life (M-QoL-HK) questionnaire will be used to measure Quality of Life among Hong Kong adults. Through a 10-point Likert Scale of 17 items, M-QoL measures four dimensions, including physical health, psychological/mental health, spiritual well-being, and overall quality of life. The items in M-QoL are considered applicable to both elderly people, palliative care patients, and caregivers by authors in the validated context of Hong Kong. ${ }^{20}$

\section{Phase 2 measurement}

The questionnaire for family caregivers contains eight parts: (A) Their relationship with the elderly person. (B) Healthcare experiences and attitudes, and preferences for EoLC. (C) Expectations of EoLC. (D) Advanced Care Planning experiences and attitudes. (E) awareness, experience, and attitudes toward Advance Directives. (F) Ideal terminal/dying situations (preferences with respect to places of care and eventual death). (G) Quality of Life; and $(\mathrm{H})$ supplementary information on demographics, financial support for caregiving, bereavement experience, and self-rated health (personal profile). The contents are similar to the elderly survey version with slight changes in wording to include an item that measures the caregivers' understanding of the elderly person's own perspective. Most of the questions are closed-ended, while there are open-ended questions for the caregivers to express their own opinions.

For both versions of surveys, there is a small ending section where interview staff can write their observation notes on respondents' emotions. Interviewers may also mark any words such as 'euthanasia' or 'hastened death' if these are mentioned during the conversations. ${ }^{21}$

Completion time for the interview is estimated to be 30-45 min. The RA will read out and explain all survey items one by one to the respondents to ensure the quality of the data collection. Furthermore, the respondents are not allowed to change their answer once the questions have been answered.

\section{Patient and public involvement (PPI) statement}

Potential barriers to study this population with PPI include the challenges of engaging the local elderly home management given that service providers are motivated to protect their residents and may hesitate before to aid research, especially without formal ethical approval. The survey was developed after consultation with public representatives, such as experienced staff members who work with the target population. We did not directly involve elderly people at the initial stage of this study as we did not have any pre-established partnership with local RCHEs. The revision of the survey content based on pilot responses took the elderly participants' feedback into account. We radically revised the survey content by deleting some awareness items, changing the wording to make the current survey easier to use in a formal study. Furthermore, we plan to disseminate the study findings in plain language in elderly homes and to the general public in the future, and their responses to the findings can influence the development of EoLC policy and practice in Hong Kong further.

\section{Data analysis}

IBM SPSS Amos 26 and Mplus Version 7 will be used for the statistical analyses. Descriptive analysis will first be conducted on demographics. Response frequencies for each measure will be tabulated and the corresponding proportions will be calculated by dividing the frequency by the number of respondents. We plan to apply Logistic Regression Analysis to investigate independent predictors of our outcomes of interests, for example, the participants' preference to have communication on ACP for their own terminal care. The associations of sociodemographic characteristics, frailty, depression and functioning status with the outcomes will be analysed.

We will apply Kappa Statistics to examine paired results between the frail elderly persons and their family caregivers. Preferences for life-sustaining treatment will be expressed in $3 \times 2$ tables and described in terms of percentage agreement and the kappa coefficient. The 
level of agreement will be described using indices such as 'agreement index' developed in the literature on treatment preferences. Where a caregiver could accurately interpret an elderly participant's preference in LST, whether that was for active treatment, against LST, or uncertain, they will gain a score of one. Thus, we will use a total score of 0 (no ability) through 8 (full ability) to know or estimate the family members' preferences in treatment for elderly residents who were at terminal stages from survey hypothetical scenarios. Combined agreement scores from all matched pairs of family caregivers and elderly respondents will represent overall agreement in the survey sample. Finally, we plan to calculate the association between elderly participants and family caregivers' characteristics and agreement scores through multiple linear regression.

\section{Ethics and dissemination}

The cross-sectional survey has been approved by the Institutional Review Board of the University of Hong Kong/ Hospital Authority Hong Kong West Cluster (IRB Reference number: UW 18-443). To respect the principles of human dignity and voluntary participation in the study, a comprehensive verbal explanation and a comprehensive information sheet will be provided to the participants (family caregivers and the frail elderly home residents) during the recruitment period, prior to the commencement of data collection. The purpose and procedure of the study, as well as the risks and benefits of participation, will be explained. Respondents will be encouraged to clarify any queries related to the study with the research staff. Oral consent will be obtained from elderly participants before commencing the individual interviews. For their family members, respondents can opt for either oral or written consent. Participation will be entirely voluntary, and respondents will be informed of their rights to withdraw or refuse to give information at any time without incurring penalties or deprivation of services.

There is a chance that these interviews may cause some stress for elderly participants beyond what is typical in their casual daily conversations given that as the survey covers death-related content. In the pilot interviews, signs of stress occasionally appeared as expected. Participants were asked whether they wanted to continue or not, and then their wishes were respected. Similarly, in the future interviews during the proposed study, the survey process will be paused in cases when participants are willing to continue despite stress to reduce potential harm to participants. The researchers will closely observe the reactions of the elderly participants. Additionally, relaxation techniques will be taught to the elderly participants on-site as needed. This will be documented in our survey and referred to staff for appropriate follow-up. Moreover, positive answers to the item on suicidal ideation item and those with depression symptoms at the cut-off score of 2 or above will be brought to the attention of the elderly home manager. ${ }^{19}$
The survey project officially began on November 1 , 2018, and we aim to complete the project by October 30,2021 . Our results of the proposed cross-sectional study will be reported with reference to the international statement in the Strengthening the Reporting of Observational studies in Epidemiology checklist for cross-sectional studies. ${ }^{22}$ The findings will be disseminated through international conference presentations and peer-reviewed academic journal publications. Meanwhile, a report in plain language will be shared with our partner elderly homes. The evidence gathered from this study has the potential to benefit research on EoLC both locally and internationally, and to further inform practitioners on the desires and the EoLC needs of the frail elderly people and their caregivers. It is very important to share the knowledge that we gain on the preferences of frail elderly home residents with professionals in healthcare sectors, such as physicians, direct service staff and the larger community. Understanding their wishes can be a great step towards developing better quality EoLC for the $8 \%$ of Hong Kong elderly population who reside in RCHEs. ${ }^{23}$

Acknowledgements We would like to thank Editage (www.editage.com) for English language editing. We would like to thank Dr. Robert Smith for reviewing an earlier version of this manuscript.

Contributors C-CL, PPHC, and BY conceptualised the survey design. BY and PPHC drafted the manuscript protocol. BY prepared the IRB application and the amendment from collaborative input. BY and XX conducted pilot survey interviews. BY and XX made the revision with C-CL. All the authors, including BY, XX, PPC, NT, $\mathrm{DC}, \mathrm{FHWC}$, and $\mathrm{C}-\mathrm{CL}$, contributed to the development of the survey protocol and approved the final version of the manuscript.

Funding The current research is supported by the Enhanced New Staff Start-up Research Grant by the University of Hong Kong (HKD 380000 for 3 years commencing November 01, 2018).

\section{Competing interests None declared.}

Patient and public involvement Patients and/or the public were involved in the design, or conduct, or reporting, or dissemination plans of this research. Refer to the Methods section for further details.

Patient consent for publication Not required.

Provenance and peer review Not commissioned; externally peer reviewed.

Open access This is an open access article distributed in accordance with the Creative Commons Attribution Non Commercial (CC BY-NC 4.0) license, which permits others to distribute, remix, adapt, build upon this work non-commercially, and license their derivative works on different terms, provided the original work is properly cited, appropriate credit is given, any changes made indicated, and the use is non-commercial. See: http://creativecommons.org/licenses/by-nc/4.0/.

\section{ORCID iDs}

Bo Yan http://orcid.org/0000-0002-9967-4122

Derek YT Cheung http://orcid.org/0000-0002-5850-5349

\section{REFERENCES}

1 Chung RY-N, Wong EL-Y, Kiang N, et al. Knowledge, Attitudes, and Preferences of Advance Decisions, End-of-Life Care, and Place of Care and Death in Hong Kong. A Population-Based Telephone Survey of 1067 Adults. J Am Med Dir Assoc 2017;18:367.e19-367. e27.

2 SW T. Advance Care Planning in Elderly Residents of Long-term Care Facilities and Its Related Factors [master's thesis. Taiwan: Taipei Medical University, 2016.

$3 \mathrm{Ni} \mathrm{P}$, Zhou J, Wang ZX, et al. Advance Directive and end-of-life care preferences among nursing home residents in Wuhan, China: a cross-sectional study. J Am Med Dir Assoc 2014;15:751-6. 
4 Wang C-W, Chan CLW. End-Of-Life care research in Hong Kong: a systematic review of peer-reviewed publications. Palliat Support Care 2015;13:1711-20.

5 Lee MC, Hinderer KA, Kehl KA. A systematic review of advance directives and advance care planning in Chinese people from eastern and Western cultures. Journal of Hospice \& Palliative Nursing 2014:16:75-85.

6 Mok E, Ting FH, Lau K-po. Advance directives and life-sustaining treatment: informed attitudes of Hong Kong Chinese elders with chronic disease. J Nurs Healthc Chronic Illn 2010;2:313-9.

7 Morley JE, Haren MT, Rolland Y, et al. Frailty. Med Clin North Am 2006;90:837-47.

8 Chu L-W, Luk JKH, Hui E, et al. Advance Directive and end-of-life care preferences among Chinese nursing home residents in Hong Kong. J Am Med Dir Assoc 2011;12:143-52.

9 Luk JKH, Chan FHW. End-Of-Life care for advanced dementia patients in residential care home-a Hong Kong perspective. Ann Palliat Med 2018;7:359-64.

10 Policies and measures on elderly care services. Legislative Council (Hong Kong): Council business division 2, 2016: 15-16.

11 Measuring the Age-friendliness of Cities: A Guide to Use Core Indicators. World Health organization (Japan): who Kobe centre, 2015: 28.

12 Chan HYL, Pang SMC. Quality of life concerns and end-of-life care preferences of aged persons in long-term care facilities. J Clin Nurs 2007;16:2158-66.

13 Russ AJ, Kaufman SR. Family perceptions of prognosis, silence, and the "suddenness" of death. Cult Med Psychiatry 2005;29:103-23.
14 Winter L, Parks SM. Family discord and proxy decision makers' endof-life treatment decisions. J Palliat Med 2008;11:1109-14.

15 Woo J, Yu R, Wong M, et al. Frailty screening in the community using the frail scale. J Am Med Dir Assoc 2015;16:412-9.

16 Chu LW, Pei KW, MH H, et al. Validation of the abbreviated mental test (Hong Kong version) in the elderly medical patient. Hong Kong Med J 1995;1:207-11.

17 Kim J, Shin M-S, Park YM, et al. Associations of advance Directive knowledge, attitudes, and barriers/benefits with preferences for advance treatment directives among patients with heart failure and their caregivers. J Card Fail 2020;26:61-9.

18 Collin C, Wade DT, Davies S, et al. The Barthel ADL index: a reliability study. Int Disabil Stud 1988;10:61-3.

19 Cheng S-T, Yu ECS, Lee SY, et al. The geriatric depression scale as a screening tool for depression and suicide ideation: a replication and extention. Am J Geriatr Psychiatry 2010;18:256-65.

20 Lo RS, Woo J, Zhoc KC, et al. Cross-Cultural validation of the McGill quality of life questionnaire in Hong Kong Chinese. Palliat Med 2001;15:387-97.

21 Wang Y-C, Lin C-C. Spiritual well-being may reduce the negative impacts of cancer symptoms on the quality of life and the desire for hastened death in terminally ill cancer patients. Cancer Nurs 2016;39:E43-50.

22 von Elm E, Altman DG, Egger M, et al. The strengthening the reporting of observational studies in epidemiology (STROBE) statement: guidelines for reporting observational studies. Lancet 2007;370:1453-7.

23 Statistical Highlights: Residential Care for the Elderly. Legislative Council (Hong Kong): information services division 2017. 